\title{
Guided Analytics Platform for Southern Region of Tamilnadu Farmer Fraternity
}

\author{
K.Sumathi, P. Deepalakshmi, K. Nagarajan
}

\begin{abstract}
Agriculture has its role to play in our economy like any other sector but the unique role and importance is comparatively high than the other sectors. Most of the families who are farmers in our country live in rural areas and find themselves aloof from technological world. Essential agricultural support services needed to be carried out in farming activities are left unaware for them. Moreover, farmer's income and productivity can be improved through extension and advisory services which would be of greater support. With the advent of technological resources that is suitable, cost effective, user friendly and scalable for the farmers certain farming activities can be improved. This paper intends to propose a data analytic platform using modern digital technology enabling effective collaboration among farmer fraternity and peripheral partners. The platform ensures of delivering enhanced insights to the agricultural community with essential guidance and tips to improve with on high crop yield; forecast and report right time for sowing and irrigation; corrective measures to be performed during pest attack so that the farmers enrich their agricultural activities with the support of efficient decision making system.
\end{abstract}

Keywords - Machine Learning Techniques, Big Data Analytics, Decision Making System for Farmers and their ecosystem.

\section{INTRODUCTION}

As per latest home ministry statistics - rate of farmers / cultivators committing suicide reaches 6,351 in numbers in 2016 across India. It is also recorded that the farmer's suicide in Tamil Nadu has increased 18 times from 2 times between 2015 and 2016. Ministry of statistics and program implementation [1] evinces that the contribution in agriculture sector to the country's Gross Domestic Product (GDP) decreased from $54 \%$ in $1950-51$ to $15.4 \%$ in 2015 16.

Hence, the dire need for provision of timely and relevant information to farmers is to be realized.

The world is on the move to transform from normal agricultural sector towards digital agricultural sector. Farmers of today face challenges from different perspectives such that financial perspective, natural resource perspective, technological perspective, social and economic perspectives. Among all perspectives, the climatic perspective where the farmers are mostly affected and the technological resource perspective if tapped upon, the result would be a helping hand to the farmers.

Revised Manuscript Received on December 16, 2019

* Correspondence Author

K. Sumathi*, Assistant Professor, Dept of CS \& IT, Kalasalingam Academy of Research and Education,Krishnankoil, Virudhunagar,Tamilnadu,India,.Sumathirajkumar2006@gmail.co

K. Nagarajan, Principal Consultant,Tata Consultancy Services, Chennai, Tamilnadu Nag12317@gmail.com

P. Deepalakshmi, Professor, Dept. of CSE, Kalasalingam Academy of Research and Education, Krishnankoil,Virudhunagar, Tamilnadu, India,deepa.kumar@klu.ac.in
The importance of these two perspectives has been realized at this momentous juncture and the researchers have attempted in studying certain farming activities and big data usage to arrive at a solution. With the natural calamities and other environmental factors, it is becoming evident for the farmers to overcome challenges and disaster. While farming activities are concerned, in most of the cases the farmers are unaware of the technologies available that will ensure in promising crop yield. With the climatic variability and cropping decisions that would yield the farmers with best results become a question mark.

It is mandatory that farmers are required to be known about sustainable practices in their field. Among all practices used for a sustainable agricultural environment, the usage of big data in farming activities have evidenced in effective results where the beneficiaries have admitted of the same through testimonials. When the farmers are provided with the necessary advisory support system, the ultimate outcome would be in the basis of improved profitability which would become a small contribution to the country's GDP. The material consequences of societal members of big data have witnessed in promising results in various sectors, but among all the agricultural sector has been benefitting off late.

With the advent of technology, there are so many evidences of research which proves to witness the transformation from agriculture to digital agriculture [2] but also from farm to data as well. The researchers in this study thus with the agricultural data as the basis which pertains to cropping, planting, climatic variations, soil culture, etc have blended application of big data analysis in arriving at effective ecosystem catering to the need of all farming fraternity. With the evident contribution of this initiative, the farmers can overcome the challenges faced in the light of their livelihood. The potentiality of big data usage in agricultural sector is vast in today's era and hence this study contributes towards other research work pertaining to application of big data in agricultural sector in bringing in an effective eco-system. Each day in each one's life there are advances and relative challenges, but the knowledge of the technology to overcome the challenges are unaware for most of the people. Only when each one acquires the knowledge of usage of technological resources they would succeed by making effective decisions, similarly when the farmers are acquainted with the usage of technological resources for effective farming they would make appropriate decisions. This would in turn contribute towards the economic development of the individual and global or nationwide. 
This research is based on the primary data and secondary data pertaining to Indian statistics where the agricultural sector and its current level of contribution to GDP exist to a considerable level or not. Off late the contribution from agricultural sector has comparatively declined and farmer fraternity who is the prime stakeholder undergo issues leading to adverse effects. Therefore, in view of contributing towards the contribution from the agricultural sector, the researchers in this study has initiated in evolving a platform that is economical and suitable as well as cost effective at par with the technology that is intended. Hence, the filed map that is possible to evolve the end result - i.e., Guided analytics platform is as follows:

- Data collection relevant to agricultural farming

- Analysis of the data collected

- Disseminate required data for the study

- Finalize the data that is appropriate for the study

- Feed inputs based on guided analytics

- Execution of program

- Compare with data fed in and generates output

- Output is delivered in the form of messages to the farmer's hand / mobile phones

- Farmers take decisions accordingly and adopt suggestive measures

- Final result is recorded for the impact and compared with previous years.

- Improvised evidences and recorded.

By way of the suggestive roadmap the farmers may be asked for testimonials and the benefits are reaped which become the expected result of this guided analytics platform. Awareness among farmers was instilled or imparted so that they ruminate on this aspect and finally use it realizing its worth. Farmers were mentored suitably from the start of problems they faced and their relative expectations with respect to agricultural activities. In view of prior to devising the platform, the farmers issues which were identified are as follows:

1. Farmers suffer due to non availability of information and specific guidance (advisory services).

2. Low productivity per acre due to lack of mechanism and technology usage.

3. Farmers in rural areas are divorced from technology and essential agricultural support services needed to carry out farming activities were lacking.

4. Lack of effective communication among farmers, other stakeholders and agriculture marketing partners.

5. Poor agriculture marketing

With the above as the basis, the study is furthered in order to arrive at concrete solutions that suit the farmers and hence this platform is a tailor-made one which strikes the bulls eye.

\section{LITERARY REVIEW}

XuanPham and MartinStack (2017) [3] has integrated the micro- and macro-level analyses via the lenses of firms in agricultural input markets and taps the need for the agricultural stakes in taking smarter decisions. The paper also elaborately discusses about agricultural needs on forefront and the competitive environment and consideration of conventional methods in agriculture. Moreover the precision agriculture is detailed here enabling the researcher in giving a road map as to what are the considerations to be made for the study pertaining to agriculture and its transformation.

Web-portal of Microsoft News center India's showcases on certain facts that is relevant to this study in terms of cloud machine learning, satellite imagery, advanced analytics and etc. The most appropriate fact is that the yield increase of $30 \%$ due to AI based advisory system for the farmers in Andra Pradesh and Karnataka. This advisory system ensured that the farmers were either intimated or alarmed through SMS in their vernacular languages on when to sow their groundnut crops and other measures that the farmers need to take from time to time. The portal also highlights of predictions on pest attacks enabling the farmers to plan accordingly. The testimonials of farmers who have been the beneficiaries have also been evidenced in this portal. In addition Microsoft has proposed the forecasting model suggested for policy makers with the help of predictive analysis for the agricultural commodities.

Kelly Bronson \& Irena Knezevic (2016) [4] in "Big Data in food and agriculture", explores the advent of big data usage and implications and relationships between various players in food and agricultural sector. In this study, the agricultural fraternity is targeted but with the evidences of this review, the extent of beneficiaries would be players from food sectors too.

Liakos KG et al. (2018) [5] review paper presents in detail the applications pertaining to machine learning in yield prediction, disease detection, weed detection, etc which is also the aspects of farming activity dealt with in this research study by the researchers. The beneficiaries of agricultural sector through the machine learning technologies and artificial intelligence based programs that provide inputs to the farmers for effective decision support system are also dealt with which is once again the focus of this study.

Savvas Dimitriadis and Christos Goumopoulos,(2008) [6] in their conference paper has published on the ways and means of automatically extracting the new knowledge on farming activities into generalized forms of rules for effective decision making. This paper also highlights that the suggestions from the study would lead to the best administration of the resources available - especially with water. They have also suggested a model of machine learning keeping inductive and iterative process as the basis of the paper. The results of the study impacts in reducing water stress in plants and effective decision pattern for the farming fraternity. Similar in this study, the researchers have kept the basis as the farming activity and have attempted to suggest a model of data analytics platform resulting in beneficial aspects of farmers.

Tim Sparapani (2017) [7] in his contributions on an Forbes article shares about the loss due to wastage in various stages of farming - harvesting, planting, water using and trucking among American farmers. The concerning fact of loss in food supply and the desperate need to draw a solution for the same. In order to address the issues the big data, RFID, sensor and other technological usages are debated elaborately. More 
importantly the precision farming tools and their usage outcome as a tech solution is effective or not is postulated. In this study, usage of big data is of prime importance while showcasing the solution to the farming issues faced by farmers.

A literary work from Agricultural systems by Sjaak. Wolfert et al (2017) [8] postulates on the impact of smart farming and the supply chain value. With the help of sensors and the smart farming practices and it leading to the change in roles, power and responsibilities of the farmer fraternity and the governance as such is discussed. In this study, the proposed platform is on the basis of big data analytics which is relevant of the study undertaken and this paves a way in contributing towards the smart farming field and towards the governance as well. The authors also highlights that the smart farming is ensured through big data and major shift transformation happens through it and contributes ultimately to the ownership, data security and privacy respectively. The related socio-economic challenges and the application beyond mere or beyond production is also evidenced in the study.

Article on "The Big Data Analytics Revolution in Agriculture" [9] evinces all with the entry of big data in agriculture also apart from big industrial sector. It explains of precision agriculture where acquisitions of data points and sharing with the help of internet connection and analyzing the agricultural data and arriving at decision support systems.

Similarly, P.J.Thorburn \& S.N.Wilkinson (2013) [10] highlights of a conceptual framework that improvise agricultural management practice for an effective ecosystem. The combination of chemical requirement is also being discussed in this paper. In this paper the testing of water quality and arriving at measures are suggested in the form of conceptual framework. With the same basis, the farming needs are taken as the basis in developing the platform by the researchers.

NirKshetri (2014) [11] in their article "The emerging role of Big Data in key development issues: Opportunities, challenges, and concerns", attempts to review academic literature pertaining to the trends in Big Data utilization and key developmental issues. The usage especially with respect to agricultural sector in farming activities in developing countries and assess the capabilities that is required at varied stages of big data analysis. In this study the data drawn from the agricultural farming activities also involve assessing the need for various capabilities in big data analysis.

\section{A. Machine Learning in Agriculture}

Agriculture plays a mammoth role in growing countries. Advanced Machine learning techniques has been used in conjunction with high speed computing and big data technologies so that it can generate new opening for data intensive science in the multi-disciplinary agriculture technologies domain. Nowadays advanced machine learning techniques are used for i) crop health and monitoring including applications such as predicting yield and crop quality, detecting disease and weed, and recognizing species (ii) water management (iii) soil management (iv) livestock management, including applications such as animal welfare and livestock production (v) Automated irrigation System (vi) drones for crop spraying and (vii) face recognition system for domestic cattle.

The filtering and classification techniques plays vital role in Agri-marketing. This paper also demonstrate how agriculture field gets benefited from machine learning technologies. Sensors also play a good role in agriculture. The sensors installed on the field sending data regarding weather forecast, soil parameter values etc., continuously. Machine learning algorithms are applied to sensor data to develop decision support system which provide farmers with necessary insights and rich guidance for farmer decision support. Liakos KG et al[16] proposed a review paper which demonstrates the importance and usage of machine learning in agriculture domain.

\section{B. Need for Bigdata Analytics in Agriculture}

The big data phenomenon is based on three dimensions such that volume, velocity and variety as indicated by Steve Sonka (2014). While we analyze the data from each dimension, there seem to present challenges but it leads in exploiting opportunities to business decisions. The decisions vary from one to another depending on the type of data available. The role of big data in various sectors is evident and when it comes to agricultural sector it has prime importance as it results in impacting or contributing towards the nation's GDP directly. Big data is represented in the form which is beyond numbers, those examples which are relevant to this study is that climatic tracking system, GPS locations, temperature, humidity, soil culture and moisture level, conversions on cell phones, etc. With these data which would be evident in analyzing the crop yield is kept as the basis of the study.

\section{PROPOSED SYSTEM}

This Proposed system enables effective collaboration between farmers and peripheral partners, offering timely decisions such as marketing, irrigation, crop monitoring, harvesting and pest control and sowing. This platform takes real time data from different sensing devices such as moisture control, wind influence, weather conditions, forecasting and soil internal parameters and to offer personalized advisory services using communication devices like mobile phones. This system is provided to farmers bundled with ICT-based benefits. It ensures in connecting the farmers with the stakeholders of multiple services through mobile phone. It can also integrate wireless sensors and cloud technologies with communication devices to provide better solution. This system will ensure necessary insights which would reach on time to farmers directly and also alert signals that can help farmers with appropriate guidance and directions.

This system would be able to 1) facilitate farmer's enrolment into the ecosystems, by asking set of standard questions and information capturing around location, area, farm and other associated information centers 2) facilitate partner ecosystem members such as weather forecast, pesticide vendors, irrigation partners, workforce providers enrolment into the system with their details, constraints and limitations 3) use cloud based 
computation model and establish network connectivity to collect data from installed sensors and other publically available information of interest 4) develop and host machine learning models that could crunch data through feature engineering and deliver enhanced insights that are useful to the communities, such as guidance and tips to farmers for crops high yield, right time for sowing and harvesting 5) suggest right actions to perform during pest attack and tips to manage drought conditions 6) offer alerts to other participating partners to stack up pesticides or Agriequipments based on weather forecast and nature of crops 7) share the appropriate harvesting time with Agri marketing partner to project good purchase price and 8) deliver all these solution in a real time anywhere anytime consumption enabled mobile devices.

To be precise, a data analytics platform will be built for agrarian community that includes maps and data regarding Weather Forecast, Precipitation, Electrical conductivity, Soil Moisture content, Wind Flow, Government policies, Nutrient contents and $\mathrm{pH}$ level, Stakeholder, Commodity prices, details of farmers and other partners of the ecosystem. For enabling this platform, big data analytics, cloud, and advanced machine learning techniques are adopted to guide farmers with high precision farming decisions, preparing support partners with necessary alerts to help farmers at right time, facilitate farm products to get sold at possible high price, delivering all these capabilities in a fingertip of farmers through mobile devices

Data Analytics Platform is built with the advent of technological resources that is suitable, scalable, user friendly and cost effective. The proposed platform will be able to 1) capture data from Farmers, other Stakeholders, National Information Centers (NICs), various Sensors mounted on the field through deployed gateways and Base stations, and to store the captured data in cloud storage offered by either Microsoft Azure or Google Cloud Platform, and use their inbuilt toolsets for integration, analysis and visualization. 2) Preprocess the data collected from various heterogeneous sources by eliminating noisy, irrelevant data and to prepare the relevant data suitable for analysis using Python and Google Tensor flow. 3) Build machine learning models using advanced machine learning techniques, big data analytics and cloud computing to analyze facts and obtain the relationship between facts and attributes. 4) deliver enhanced insights that are useful to the farmer communities, such as guidance and tips to farmers for crops high yield, right time for sowing and irrigation, etc., through communication devices such as mobile phones, etc., 5) design various Application Programming Interface such as Farmers API, Partners API and Open Data Services for effective collaboration within the farmer ecosystem and to enhance social, economic status of farmers and agriculture production in rural area.

\section{A. Components of Guided Analytics System}

The presented system relates to data analytics particularly to a computer implementable system and method for data analytics catering to the need of the agriculture society. The guided analytics system consists of three components such as processors, interfaces and memory joined with processor. Computer readable instructions are fetched and executed by the processors. The interfaces include hardware and software interfaces. The memory includes three layers such as Consumption Layer, Data Layer and Functional Layers. The Various Components of Guided Analytics System is showcased in Fig. 1.

Consumption layer: This layer offers necessary Application Programming Interface for farmers and Peripheral Partners, Open Data Services which can be accessed by any one across India for effective collaboration within the farmer ecosystem.

Farmers API : It is responsible for 1) Facilitating farmer's enrolment into the ecosystems 2) Distributing advisory services to farmers such as right actions to be perform during pest attack and tips to manage drought conditions 3 ) Delivering all these solution in a real time anywhere anytime consumption enabled mobile devices.

Peripheral API : This API is responsible for 1) Facilitating partner ecosystem such as weather forecast, pesticide vendors, irrigation partners, workforce providers enrolment into the system with their details, constraints and limitations 2) Offering alerts to other participating partners to stack up pesticides or agri-equipments based on weather forecast and nature of crops.

Open Data Services: Using this, farmers can share the appropriate harvesting time with agri-marketing partner to project good purchase price and eliminate middleman culture in agriculture field

Data layer: This layer is responsible for acquiring data from the data sources and, if necessary, converting it to a

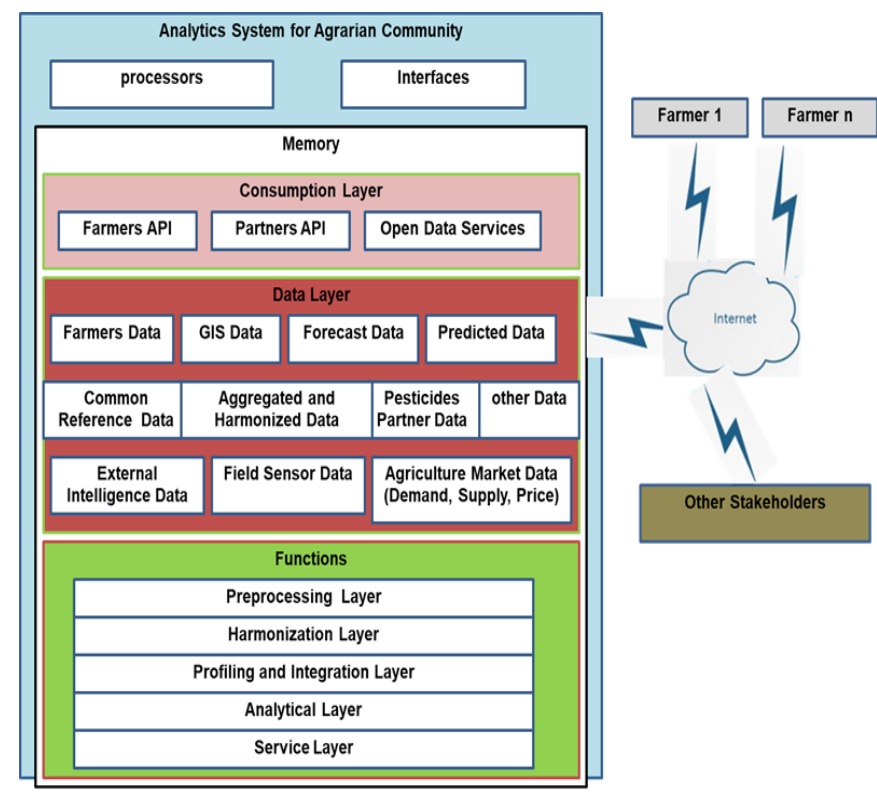

Fig.1: Components of Guided Analytics System

format that suits how the data is to be analyzed. This layer will also perform necessary data quality and profiling exercises to improve the data trust worthiness. It also performs necessary integrations across different data sources to deliver and unified data needed for machine learning algorithms.

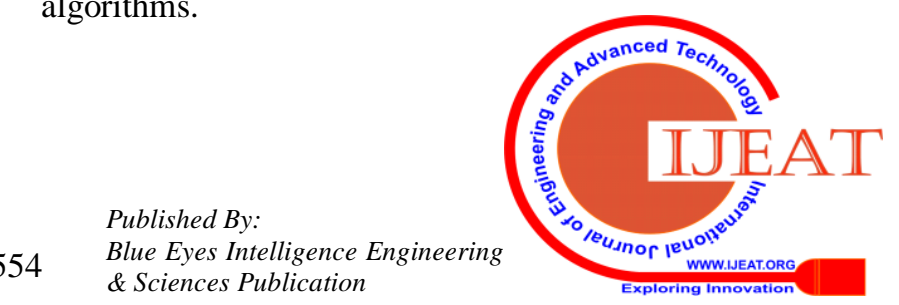


Functional layers: Functional Layers offer different operations needed to realize the solution in a modular way. It consists of 5 layers each one have very specific objective function starting from data observation still it delivers all necessary insights to the eco system.

Preprocessing, Harmonizing, Profiling and Integration Layers, Service Layer : Preprocessing is done by removing noisy and irrelevant data. Required data is extracted from agriculture and external intelligent dataset. The extracted data is standardized into a format suitable for further processing. Normalization, Machine Learning algorithms, Cognitive computing, and benchmarking solutions might be used for Data Transformation and Analytics. Python and Google tensor flow is adopted to consolidate and integrate the data. Cloud-based wireless platforms, linked open data are being used for data transfer. Service Layer provides necessary services to farmers, peripheral partners and public.

Analytical layer: The analytics layer uses set of machine learning algorithm that would be getting executed for specific objective functions such as optimized irrigation schedule, understanding the plant infection (disease) using computer vision algorithms, predicting best pest response decisions using deep learning techniques. All these algorithms in this layer are appropriately governed and recalibrated at regular intervals.

\section{B. Methodology}

This research work proposes a data analytics platform which is built with a leveraging effect on modern digital technologies to create a positive impact to farmer fraternity by bringing all supporting elements in one place and offer insights throughout the crop cultivation. The proposed data analytics platform consists of 5 phases (5Cs) including Capturing, Consolidation, Contextualize, Consume and Collaborate.

Capturing: Data can be collected from the Farmers, other Stakeholders, various Sensors in the fields, National Information Centers (NICs). Data can be collected from farmers by asking set of standard questions and information capturing around location, area, farm and other associated information centers. Sensors mounted on crop/standalone place above and below ground level can capture data about soil moisture, soil temperature, wind direction, solar radiation, leaf wetness and greenness, rainfall level, etc., These sensors can be used in conjunction with a Global Positioning System (GPS). Data from weather forecast, pesticide vendors, irrigation partners, workforce providers are also collected and stored. Data can be collected through base stations and appropriate Application Programming Interface (APIs) and stored in cloud.

Consolidation: Preprocessing is done by removing noisy and irrelevant data. Required Data is extracted from agriculture, external intelligent dataset and standardized into a format suitable for further processing. Python and Google tensor flow will be adopted to consolidate and integrate the data. Cloud-based wireless platforms, linked open data will be used for data transfer.

Contextualize: Machine learning models are developed and hosted that could crunch data through feature engineering. For realizing this analytics platform, big data analytics, cloud, and advanced machine learning techniques are adopted to guide the farmers. Normalization, Machine Learning algorithms, Cognitive computing, and benchmarking solutions might be used for Data Transformation and Analytics.

Consume : Machine learning models will be capable of delivering enhanced insights that are useful to the farmer communities, such as guidance and tips to farmers for crops high yield, right time for sowing and harvesting, irrigation, right actions to be performed during pest attack, etc., The Framework of Guided Analytics System (GAS) is showcased in Fig.2.

Collaborate: The system receives analysis results and presents them to the appropriate output layer. The system offers farmers API, Partners API and opens data services for effective collaboration within the farmer ecosystem. Adequate security measures and data privacy policy will be imposed while sharing the data within the ecosystem. It will also anonymize the data that would be helpful for government programs and formulating policies for farmers. It can be challenging to visualize the outcome of the analytical layer. Data Visualization techniques are used to present the outcome of the analytical layer that will be useful for Agriculture Marketing.

The system offers alerts to other participating partners to stack up pesticides or agri-equipments based on weather forecast and nature of crops, share the appropriate harvesting time with Agri marketing partner to project good purchase price and deliver all these solution in a real time anywhere anytime consumption enabled mobile devices.

\section{IMPLEMENTATION}

A model is structured to provide personalized advisory services to the farmers. This model can also integrate wireless sensors and script technology with communication devices to provide better solutions for farmers. Different kind of sensors like Electromagnetic, Optical, Mechanical, Electrochemical, Airflow, Acoustic, Dielectric Soil Moisture and Location Sensors, etc., can be mounted in the field. Base station system which has enough hardware capacities to process the numerous sensing data from field sensors and support various network technologies such as Ethernet, Bluetooth and WLAN can be installed and tested.

Real time data can be captured from different sensing devices such as moisture control, wind influence, weather forecasting and soil internal parameters, etc., through Base stations installed in the field. Data is collected from 1) farmers using questionnaire or API 2) National Information Centers, and other stakeholders through appropriate APIs The relevant data is extracted and converted into the required formats suitable for further processing. Measures can be taken to determine patterns in soil and weather conditions etc. The Entire Framework of Guided Analytics System is shown in Fig. 2.

The system and method for data analysis of agrarian community is disclosed. The system comprises a registration module, which is configured to register one or more users. The system is configured to receive request from one or more users for data analysis in 
the Agrarian Community. The system comprises a data preprocessing module, which is configured to retrieve data from multiple heterogeneous resources. The resources are farmer's data, external intelligence data, sensor data, pesticide partner's data, predicted data, agriculture market data.

The data pre-processing module is also configured to pre-processes the data for better analysis. The data preprocessing module is also configured to unify data from heterogeneous information. The system comprises an analysis module, which is configured to analyze the preprocessed data. The analysis module is configured to calculate one or more weightage factors. The analysis module is configured to apply correlation rules on the preprocessed data. The system comprises a data identification The system comprises a generation module, which is configured to generate results based on analysis of the data. The generation module is also configured to rectify errors and outliers in the result data. In one implementation, the method includes acquiring farmer's data, peripheral partners' data and other public data, and process the data to obtain required results which are measures and attributes.

The method also analyzes facts and obtains the relationship between facts and attributes. The method also includes identifying reference links, where each of the links is investigative of a relationship between the facts and the attributes. The method leverages one or more analytical models in the catalog to establish relationships, correlations and deep integrated insights that would be useful for agronomy community partners. The data flow diagram of Guided Analytics System (GAS) is given in Fig. 3.

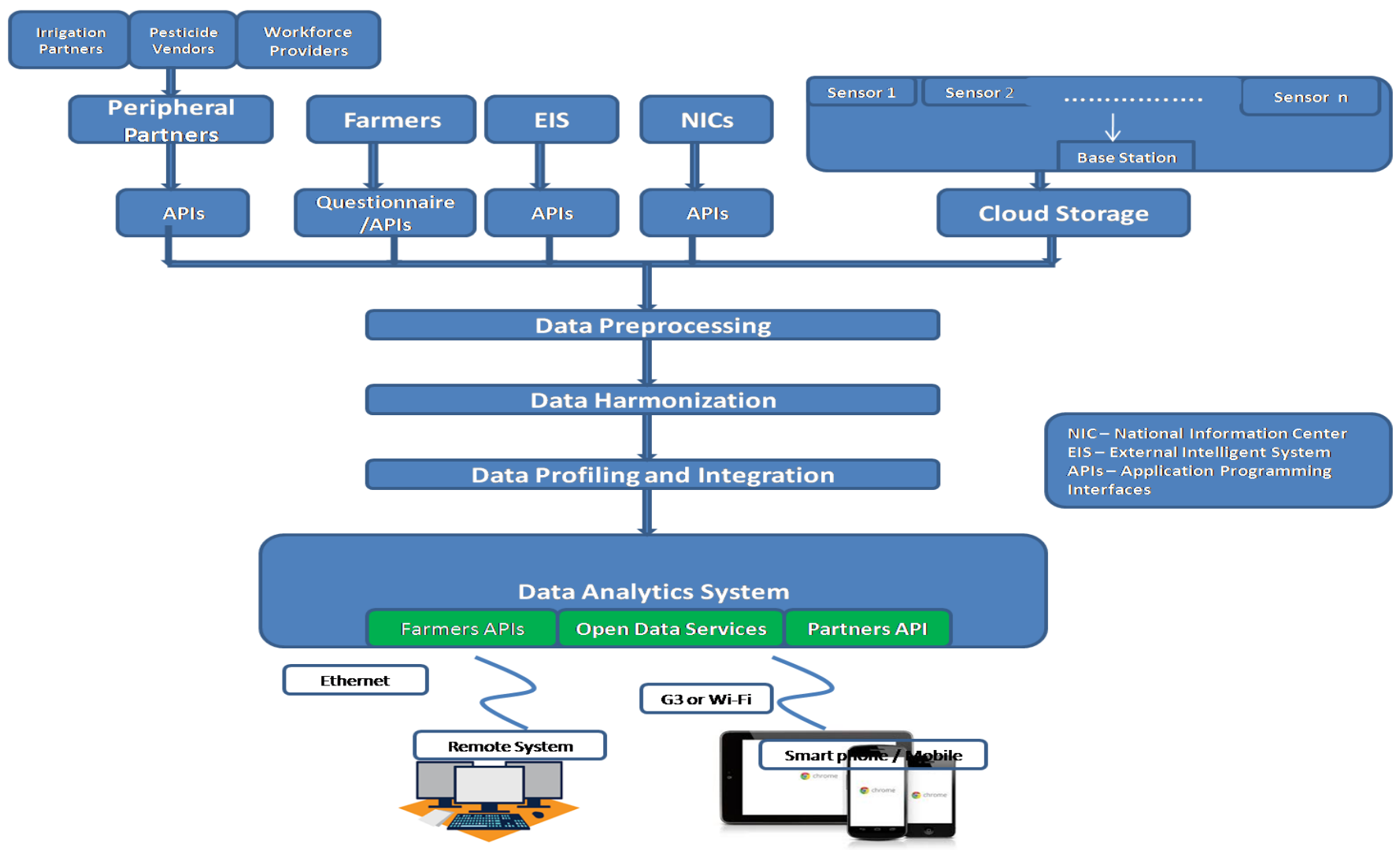

Figure 2: Framework of Guided Analytics System

This system can be provided to formers with the benefits of ICT. It connects farmers with the various stakeholders of multiple services through mobile phone. System will also receive various requirements of farmers and other stakeholders and sends the response on time. Feedback can also be collected from farmers and other stakeholders for further enhancement of the Analytics system. Feedback collected from the stakeholders will be analyzed so that the designed model can be upgraded to meet the requirements of primary and other stakeholders. Results of feedback analysis will be incorporated in the designed model.

\section{RESULTS AND DISCUSSIONS}

This collaborative extended big data analytics platform will deliver the following benefits to farmer ecosystems as well as partners.

1. Enhance farmer awareness of market demand and crop selection insights considering various parameters such as season, soil conditions, weather forecasting and demand

of produce in the market

2. Aid with appropriate insights to prepare the land for proper cultivation starting from ploughing, seed varieties, cropping procedures, growth related advise, pest control, type of irrigation methods for effective moisture control, crop harvesting and

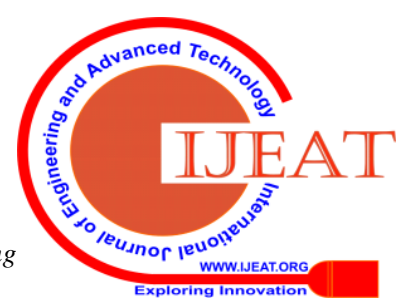


preparing for the market, right advise for maximum profit selling

3. Sustainability aspect in terms of optimizing crop spacing, watering techniques and moisture control, advice of reusability of water, resources and plant produces

4. Partners to benefit are pesticides and agro equipment service providers, they can get appropriate forecast and leverage optimization techniques to manage their resources and workforce

5. Insights to government to span out research on plant protection initiatives, sustainability, price allocation for produce, insurance for corps and in case of disaster, estimating the impacts are some of the direct use of data to arrive at informed decisions.

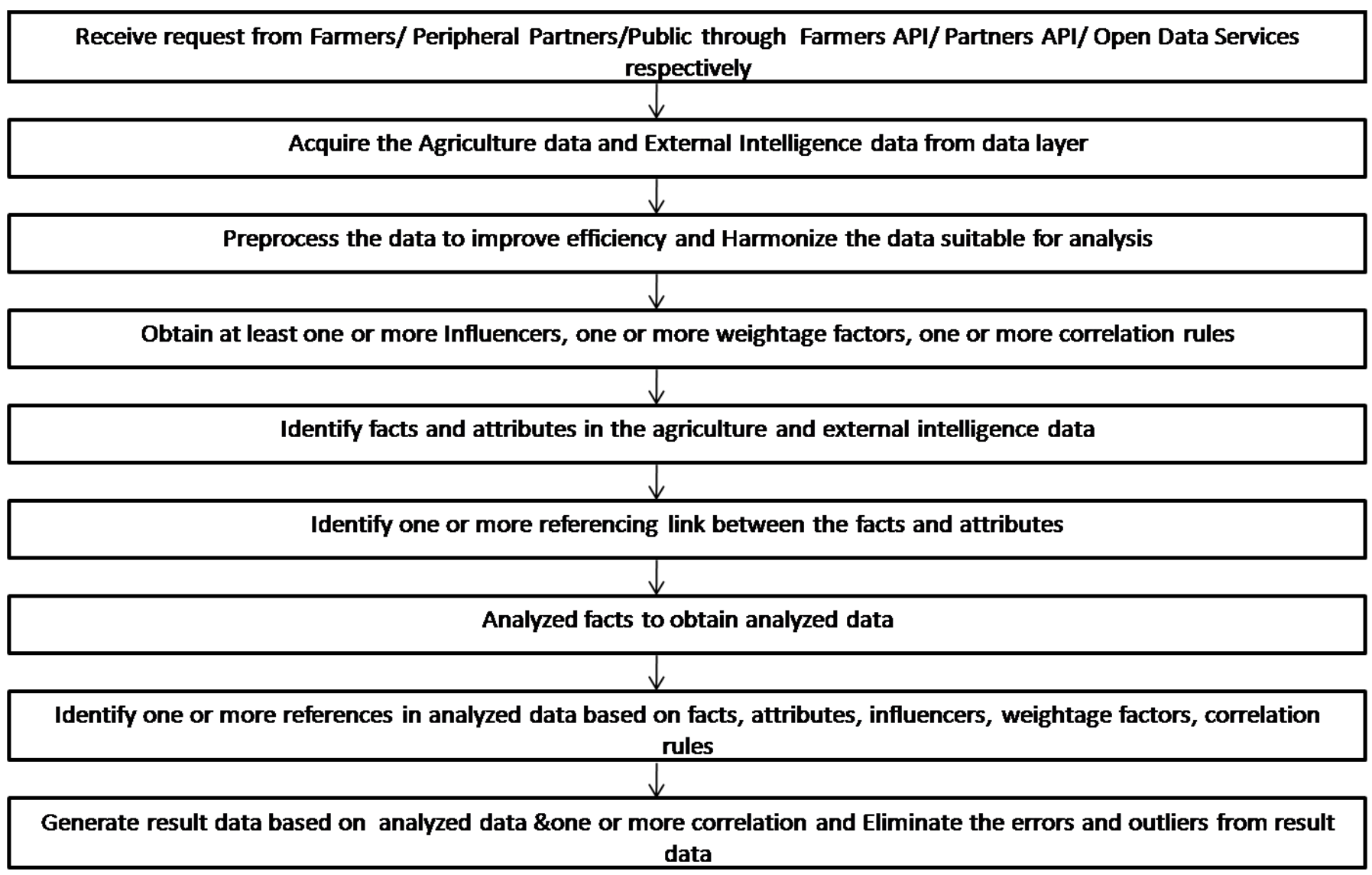

Fig 3 : Data Flow Diagram of Guided Analytics System

Soil moisture, air temperature, light and fertilizer level of the soil are monitored frequently through field sensors. Fig.4 (a-f) show distribution of fertilizer level, fertilizer alert, soil moisture, irrigation alert, light values and air temperature of the tested area for 3 months from June to August of 2018.

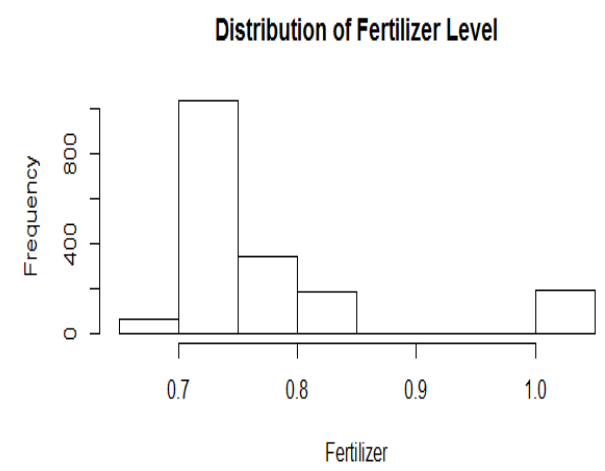

Fig.4.a Distribution of Fertilizer Level

The fertilizer alert is sent to farmers when the level of the soil fertilizer level is lower than the threshold value. The
Fertilizer alert with recommendations based on the crop type, soil type are sent to the stakeholders

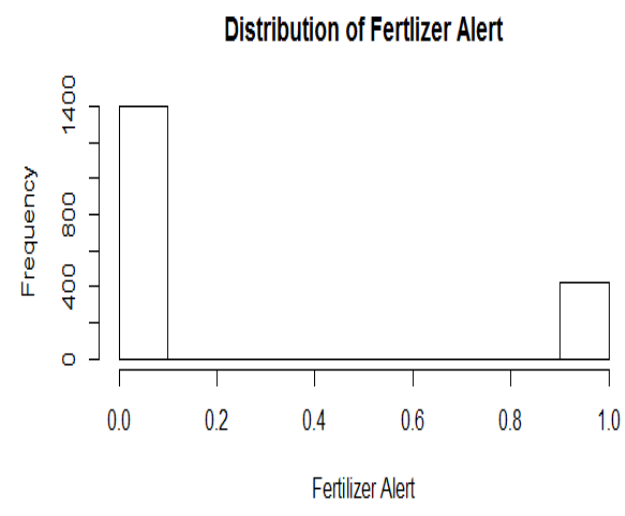

Fig.4.b Distribution of Fertilizer alert to stakeholders based on fertilizer level of the soil 


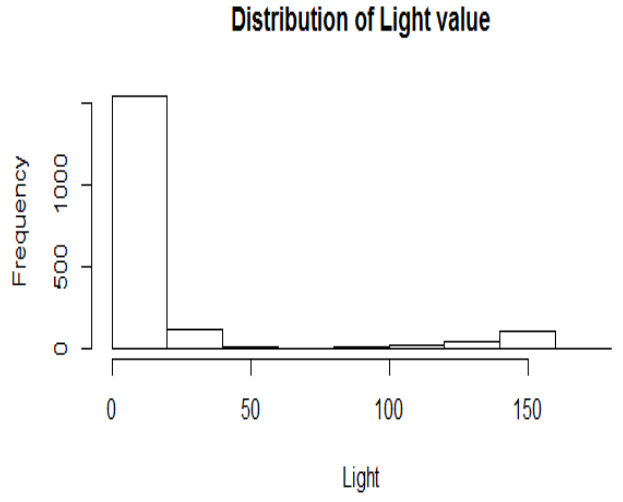

Fig.4.c Distribution of Light

The value of soil moisture plays a vital role in irrigation alert system. Weather forecast information is monitored when the soil moisture content goes below the threshold value which is varied from crop to crop.

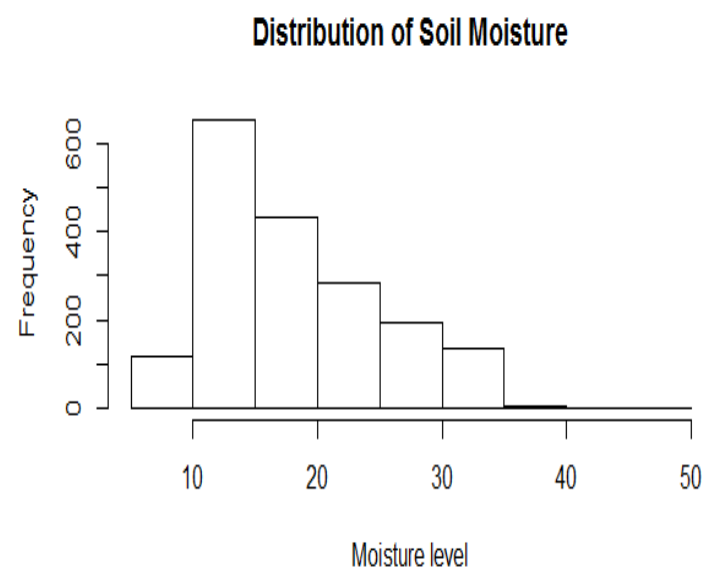

Fig.4.d Distribution of Soil Moisture

The necessary irrigation alert is sent to the farmers if weather prediction says "no" for next 24 to 48 hours. Soil moisture is analyzed before sending the fertilizer alert also.

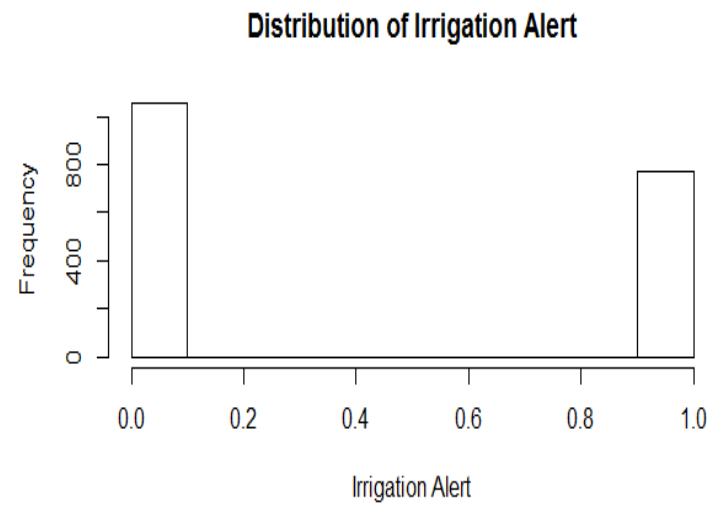

Fig.4.e Distribution of Irrigation alert to stakeholders based on soil moisture and weather forecast

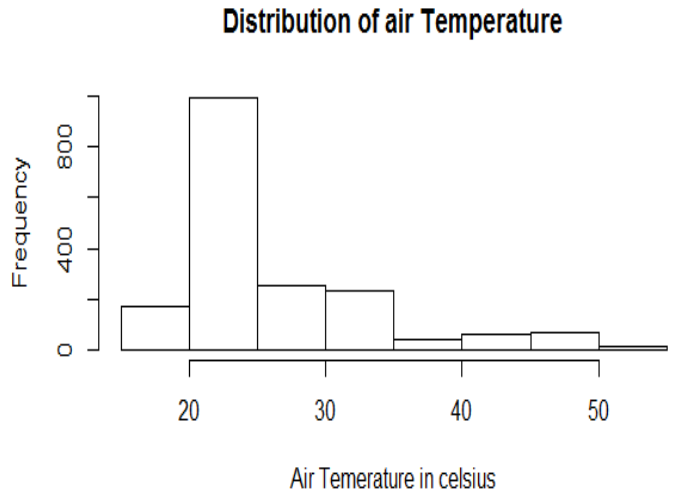

Fig.4.f Distribution of air temperature

Weather forecast details are checked based on the soil moisture values which are taken from field sensors and necessary alerts sent to the stake holders.

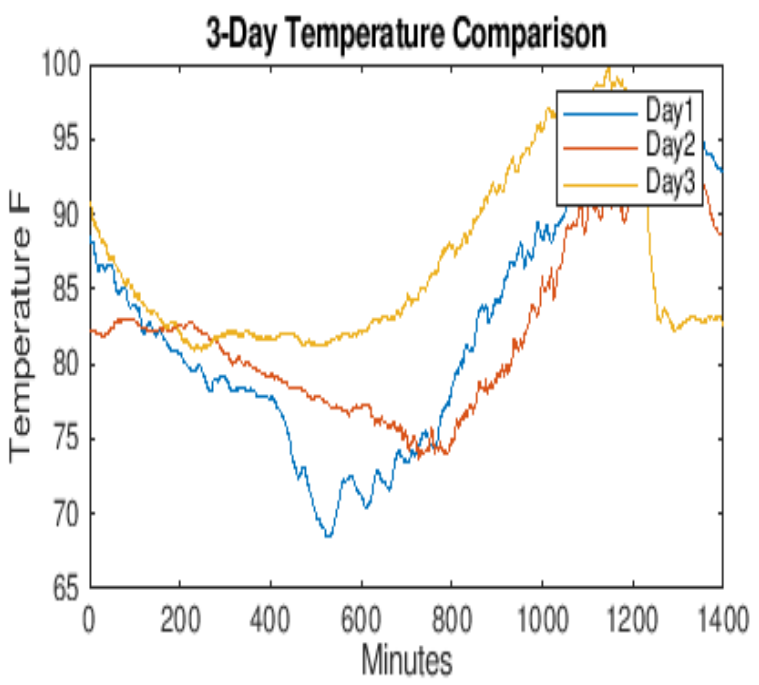

Fig.5.a Temperature Analysis for 3 days

Fig.5.a shows that temperature comparison of past three days; histogram of temperature variation is shown in Fig. 5.b. Fig.5.c shows the temperature and wind speed analysis.

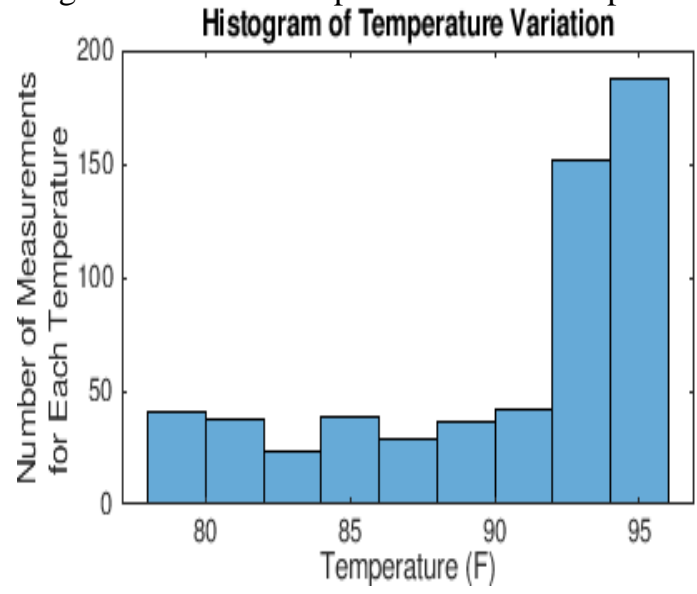

Fig.5.b. Temperature Variation 


\section{CONCLUSION AND FUTUREWORK}

This paper proposes a Data Analytics Platform for Agrarian community and their stakeholders catering especially to the southern Tamil Nadu region. . Using this platform, Farmers in rural sectors can get personalized advisory services such as crop monitoring, pesticides, harvesting, sowing seeds based on soil internal characteristics, irrigation tips through the proposed Guided Analytics. Farmers can enhance the farming activities with the help of efficient decision making system. Support partners send necessary alerts to help farmers at right time.

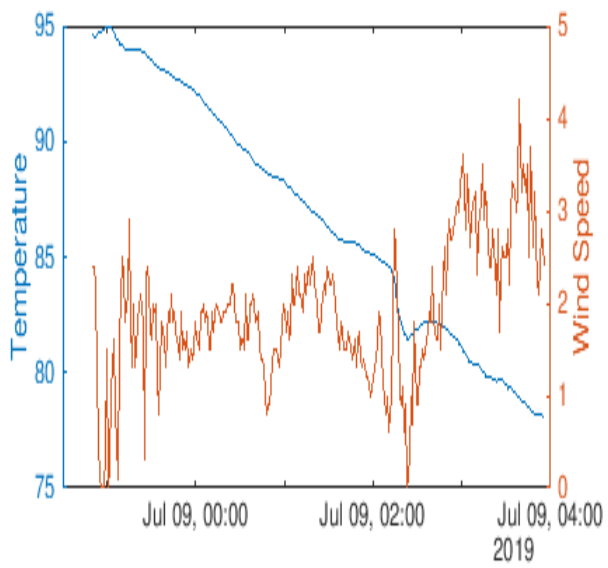

Fig.5.c. Temperature and wind speed analysis

The effective collaboration among farmers and ecosystem partners will be enabled for services and guidance. Middle man culture in the agriculture field is eliminated and ensures harmony among farmers and their lives. The farmers are provided with high precision decisions and actions for maximum crop productivity and good offers to sell their produce at higher rates. Intelligent use of irrigation can be achieved by identifying soil moisture contents using geographical maps, weather forecasting and intelligence gather through sensors deployed.

In future, this system can act as a prediction system which will give recommendations like guidance to the farmers for direct sale/ hold, wait then sale, suggesting the place where to sale the harvested crop, tips to farmers regarding sowing seeds based on, Internal characteristics of the soil and marketing needs by analyzing the history of previous year agri- data and current needs. With the basis of this study, future researchers can develop prediction system that will ensure in resolving farmer's issue.

\section{REFERENCE}

1. Robert J.McQueenaStephen, R.GarnerbCraig,G.Nevill-ManningbIan and H.Wittenb (2005), "Applying machine learning to agricultural data" , Computers and Electronics in Agriculture, Vol:12 (4), Pp: 275-293

2. Liakos KG, Busato P, MoshouD,Pearson S, Bochtis D.(2018), "Machine Learning in Agriculture: A Review", Sensors (Basel), August, Vol:18(8). pii: E2674. doi: 10.3390/s18082674

3. SavvasDimitriadis ; Christos Goumopoulos,(2008),"Applying Machine Learning to Extract New Knowledge in Precision Agriculture Applications ",, Panhellenic Conference on Informatics ISBN: 978-0-7695-3323-0

4. Sjaak. Wolfert,Lan Ge, Cor et Marc-JeroenBogaardt (2017), "Big Data in Smart Farming - A review", Agricultural Systems, Vol: (153) May,Pp:69-80

5. Andreas Kamilaris, AndreasKartakoullis,Francesc $X$ and PrenafetaBoldú (2017), "A review on the practice of big data analysis in agriculture"', Computers and Electronics in Agriculture, Vol:(143), December Issue, Pp:23-37

6. P.J.Thorburn\&S.N.Wilkinson (2013)," Conceptual frameworks for estimating the water quality benefits of improved agricultural management practices in large catchments",Agriculture, Ecosystems \& Environment, Vol: 180(1), November Issue, Pp:192-209

7. Steve Sonka(2014),"Big Data and the Ag Sector: More than Lots of Numbers", International Food and Agribusiness Management Review, Vol:17(1)

8. Http://www.prsindia.org/uploads/media/Analytical\%20Report/State $\% 20$ of\%20Agriculture\%20in\%20India.pdf

9. https://www.sciencedirect.com/science/article/pii/S0308521X16303 754

10. https://kundoc.com/pdf-how-data-analytics-is-transformingagriculture-.html

11. https://news.microsoft.com/en-in/features/ai-agriculture-icrisat-uplindia/

12. https://www.forbes.com/sites/timsparapani/2017/03/23/how-bigdata-and-tech-will-improve-agriculture-from-farm-toable/\#17f8b4765989.

13. https://www.searchtechnologies.com/blog/big-Idata-analyticsagriculture

14. https://doi.org/10.1177/2053951714564227

15. https://doi.org/10.1177/2053951716648174

16. Liakos $\mathrm{KG}^{1}$, Busato $\mathrm{P}^{2}$, Moshou $\mathrm{D}^{3,4}$, Pearson $\mathrm{S}^{5}$, Bochtis $\mathrm{D}^{6}$.Machine Learning in Agriculture: A Review.,Sensors (Basel). 2018 Aug 14;18(8). pii: E2674. doi: 10.3390/s18082674.

17. https://ieeexplore.ieee.org/document/5565120

18. https://ieeexplore.ieee.org/. 